\title{
A HYBRID PROXIMAL POINT ALGORITHM FOR FINDING MINIMIZERS AND FIXED POINTS IN CAT(0) SPACES
}

\author{
GODWIN CHIDI UGWUNNADI, ABDUL RAHIM KHAN AND MUJAHID ABBAS
}

\begin{abstract}
We introduce a hybrid proximal point algorithm and establish its strong convergence to a common solution of a proximal point of a lower semi-continuous mapping and a fixed point of a demicontractive mapping in the framework of a $\operatorname{CAT}(0)$ space. As applications of our new result, we solve variational inequality problems for these mappings on a Hilbert space. Illustrative example is given to validate theoretical result obtained herein.
\end{abstract}

\section{INTRODUCTION}

Let $H$ be a real Hilbert space with the inner product $\langle.,$.$\rangle which induces the norm$ \|.. , and $T$ a self mapping on a nonempty, closed and convex subset $C$ of $H$. A point $z \in C$ is called a fixed point of $T$ if and only if $T z=z$. The set of all fixed points of $T$ is denoted by $F(T)$.

Recall that a mapping $T: C \rightarrow C$ is called $k$-strict pseudo-contraction (in the sense of Browder-Petryshyn [8] ) if for all $x, y \in C$, there exists $k \in[0,1)$ such that

$$
\|T x-T y\|^{2} \leq\|x-y\|^{2}+k\|(I-T) x-(I-T) y\|^{2} .
$$

Strict pseudo-contractions have many applications because of their connection with inverse strongly monotone operators. Indeed, if $A$ is a strongly monotone operator, then $T=I-A$ is a strict pseudo-contraction, where $I$ is identity mapping on $C$ and the problem of finding zeros for $A$ reduces to a fixed point problem for $T$, and vice versa $([13,47])$.

A mapping $T: C \rightarrow C$ is called nonexpansive if for all $x, y \in C$, we have

$$
\|T x-T y\| \leq\|x-y\| .
$$

Note that the class of strict pseudo-contractions includes the class of nonexpansive mappings.

The mapping $T$ is said to be demicontractive (or $k$-demicontractive) if there exists $k \in(0,1)$ such that

$$
\|T x-p\|^{2} \leq\|x-p\|^{2}+k\|T x-x\|^{2} \forall x \in C, p \in F(T) .
$$

Note that, the class of demicontractive mappings properly includes the class of quasi-nonexpansive mappings for $k \geq 0$. A mapping $T: C \rightarrow H$ is called $\gamma$-inverse strongly monotone (see [11]) if there exists a positive real number $\gamma$ such that

$$
\langle T x-T y, x-y\rangle \geq \gamma\|T x-T y\|^{2} \text { for all } x, y \in C .
$$

Key words and phrases. Proximal point, Demicontractive mapping, Fixed point, $\triangle$ convergence, Strong convergence, CAT(0) space. 2010 Mathematics Subject classification. 47H09, 47J25. 
The Mann iteration process [41] is defined by the sequence $\left\{x_{n}\right\}$ :

$$
\left\{\begin{array}{c}
x_{1}=x \in C \\
x_{n+1}=\alpha_{n} x_{n}+\left(1-\alpha_{n}\right) T x_{n}, n \geq 1
\end{array},\right.
$$

where $\left\{\alpha_{n}\right\}_{n=1}^{\infty} \subset(0,1)$.

The problem of approximating fixed points of nonexpansive mappings via the above iteration process has been extensively studied by Reich [42] (in this connection, see also [43]).

It is known [42] that if $T$ is nonexpansive on a closed and convex subset $C$ of a uniformly convex Banach space with a Frecht differentiable norm and $F(T) \neq \emptyset$, then the sequence $\left\{x_{n}\right\}$ generated by Mann iteration process converges weakly to a fixed point of $T$ provided that the control sequence $\left\{\alpha_{n}\right\}_{n=1}^{\infty}$ satisfies $\sum_{n=1}^{\infty} \alpha_{n}(1-$ $\left.\alpha_{n}\right)=\infty$. The Mann iterative algorithm also gives weak convergence in the broader setting of strict pseudocontractions [37] (see also [5]).

However, in view of the counterexample in [22], Mann iterative algorithm does not converge strongly, in general. Thus, to obtain strong convergence, one needs to modify the Mann iterative algorithm and strengthen the hypotheses on the mapping. Recently, Hussain et al. [27] obtained strong convergence of an iterative method for nonexpansive mappings. Marino et al. [36] showed that the result in [27] is also true for strict pseudo-contractions under different assumptions on the coefficients.

On the other hand, the proximal point algorithm (PPA) is an optimization method which provides a minimizer of a convex lower semicontinuous function on a Hilbert space. Its origin goes back to the work in [39] and references therein. Rockafellar [45] raised a question as to whether the PPA always converges strongly which was answered in negative by Guler [24] (in this connection see also [10] and $[5])$.

The structure of ambient space plays a vital role in solving a fixed point equation. Banach space being a vector space possesses a convex structure in a natural way. Thus geometric properties of Banach space provide a natural setting to study the existence of fixed points of certain mappings.

However, metric spaces do not have the convex structure. Ever since Takahashi [49], studied fixed points of nonexpansive mappings in the setting of convex metric spaces, different convex structures have been introduced in metric spaces. CAT(0) space is a typical example of a convex metric space and Hilbert ball with the hyperbolic metric is another important example of CAT(0) spaces ( for details, see the book by Goebel and Reich [23]). It is well known that pre-Hilbert spaces, $\mathbb{R}$-trees [7] and Euclidean buildings [9] are CAT(0) spaces. For a thorough discussion of these spaces and their role in various branches of mathematics, we refer to [7] and references therein. The study of fixed points in the setup of CAT $(0)$ spaces was initiated by Kirk $([29,30])$. He showed that every nonexpansive mapping defined on a nonempty closed, convex and bounded subset of a CAT(0) space always has a fixed point. Lim [32] introduced the notion of $\triangle$-convergence in metric spaces. Kirk and Panyanak [31] studied this concept in the framework of CAT(0) spaces and showed that many results which involve weak convergence such as Opial property and Kadec-Klee property have precise analogues in this setting. Later on, Dhompongsa and Panyanak [20] obtained $\triangle$-convergence results for the Picard, Mann, and Ishikawa algorithms in $\mathrm{CAT}(0)$ spaces. 
Recently, many algorithms for solving optimization problems in linear spaces (Euclidean spaces, Hilbert spaces, and Banach spaces) have been studied in the setup of differentiable manifolds ( $[21,33,44])$.

Bačák [4] has considered the PPA in the framework of a $\operatorname{CAT}(0)$ space $(X, d)$ as follows:

Let $f: X \rightarrow(-\infty, \infty]$ be a proper convex and lower semi-continuous function. Define a sequence $\left\{x_{n}\right\}$ by

$$
\left\{\begin{array}{c}
x_{1} \in X \\
x_{n+1}=\underset{y \in X}{\arg \min }\left(f(y)+\frac{1}{2 \lambda_{n}} d^{2}\left(y, x_{n}\right)\right),
\end{array}\right.
$$

where $\lambda_{n}>0$, and $n \geq 1$. It has been shown that if $f$ has a minimizer and $\sum_{n=1}^{\infty} \lambda_{n}=\infty$, then $\left\{x_{n}\right\}, \triangle$-converges to the minimizer of $f[3]$.

Cholamjiak et al. [15] established that if $f: X \rightarrow(-\infty, \infty]$ is a proper convex and lower semi-continuous function, where $X$ is a complete CAT( $(0)$ space. Let $T_{1}$ and $T_{2}$ be nonexpansive mappings on $X$ such that $\Omega:=F\left(T_{1}\right) \bigcap F\left(T_{2}\right) \bigcap \arg \min _{y \in X} f(y) \neq$ $\emptyset$. Define for arbitrary $x_{0} \in X$, sequence $\left\{x_{n}\right\}$ by:

$$
\left\{\begin{array}{l}
z_{n}=\underset{y \in X}{\arg \min }\left[f(y)+\frac{1}{2 \lambda_{n}} d^{2}\left(y, x_{n}\right)\right] \\
y_{n}=\left(1-\beta_{n}\right) x_{n} \oplus \beta_{n} T_{1} z_{n} \\
x_{n+1}=\left(1-\alpha_{n}\right) T_{1} x_{n} \oplus \alpha_{n} T_{2} y_{n}
\end{array}\right.
$$

where $\left\{\alpha_{n}\right\}$ and $\left\{\beta_{n}\right\}$ are sequences such that for some $a, b$, we have $0<a \leq$ $\alpha_{n}, \beta_{n} \leq b<1$ and $\left\{\lambda_{n}\right\}$ is a sequence such that $\lambda_{n} \geq \lambda>0$ for all $n \in \mathbb{N}$ and for some $\lambda$. Then $\left\{x_{n}\right\}$ strongly converges to a point in $\Omega$.

Recently Chang et al. [12] introduced the following $S$ - type iteration process to compute minimizer of a convex function and a common fixed point of asymptotically nonexpansive mappings in $\operatorname{CAT}(0)$ spaces for an arbitrary $x_{0} \in C$, let $\left\{x_{n}\right\}$ be the sequence defined by:

$$
\left\{\begin{array}{l}
z_{n}=\underset{y \in C}{\arg \min }\left[f(y)+\frac{1}{2 \lambda_{n}} d^{2}\left(y, x_{n}\right)\right] \\
y_{n}=\alpha_{n} x_{n} \oplus \beta_{n} T_{1}^{n} x_{n} \oplus \gamma_{n} T_{2}^{n} z_{n} \\
x_{n+1}=\delta_{n} T_{2}^{n} x_{n} \oplus \eta_{n} S_{1}^{n} x_{n}+\xi_{n} S_{2}^{n} y_{n}
\end{array}\right.
$$

where $\Omega:=F\left(T_{1}\right) \bigcap F\left(T_{2}\right) \bigcap F\left(S_{1}\right) \bigcap F\left(S_{2}\right) \bigcap \arg \min _{y \in C} f(y) \neq \emptyset$ and $\left\{\alpha_{n}\right\}$, $\left\{\beta_{n}\right\},\left\{\gamma_{n}\right\},\left\{\delta_{n}\right\},\left\{\eta_{n}\right\},\left\{\xi_{n}\right\}$ are sequences in $[0,1]$ with conditions $\alpha_{n}+\beta_{n}+\gamma_{n}=1$ and $\delta_{n}+\eta_{n}+\xi_{n}=1$, where $0<a \leq \alpha_{n}, \beta_{n}, \gamma_{n}, \delta_{n}, \eta_{n}, \xi_{n} \leq b<1$. They proved strong and $\triangle$-convergence of the sequence $\left\{x_{n}\right\}$ to a point in $\Omega$.

In this paper, we establish strong convergence of a hybrid proximal point algorithm to a fixed point of an $L$-Lipschitizian demicontractive mapping and minimizer of a proper convex and lower semi-continuous function defined on a nonempty closed and convex subset of a complete $\operatorname{CAT}(0)$ space, that is, we find a common solution for a fixed point of demicontractive mapping and minimizer of a proper convex and lower semi-continuous function. We also give some applications of our result. 


\section{Preliminaries}

We denote by $\mathbb{N}, \mathbb{R}^{+}, \mathbb{R}$ the set of natural numbers, the set of nonnegative real numbers and the set of real numbers, respectively.

Let $(X, d)$ be a metric space and $x, y \in X$. A geodesic path joining $x$ to $y$ (or, a geodesic from $x$ to $y$ ) is a map $\gamma:[a, b] \subseteq \mathbb{R} \rightarrow X$ such that $\gamma(a)=x, \gamma(b)=y$, and $d\left(\gamma(t), \gamma\left(t^{\prime}\right)\right)=\left|t-t^{\prime}\right|$ for all $t, t^{\prime} \in[a, b]$. In particular, $\gamma$ is an isometry and $d(x, y)=b-a$. A geodesic segment in $X$ is the image $\gamma([a, b])$ of a geodesic path in $X$ joining $x$ and $y$. A metric space $X$ is said to be a geodesic space if every two points of $X$ are joined by a geodesic, and $X$ is said to be uniquely geodesic if there is exactly one geodesic joining $x$ and $y$ for each $x, y \in X$. A geodesic segment is denoted by $[x, y]$, if it is unique.

A geodesic triangle $\triangle\left(x_{1}, x_{2}, x_{3}\right)$ in a geodesic space $X$ consists of three points $x_{1}, x_{2}, x_{3}$ of $X$, called the vertices of $\triangle\left(x_{1}, x_{2}, x_{3}\right)$ and three geodesic segments joining each pair of vertices called the edges of $\triangle\left(x_{1}, x_{2}, x_{3}\right)$. A comparison triangle of a geodesic triangle $\triangle\left(x_{1}, x_{2}, x_{3}\right)$ is the triangle $\bar{\triangle}\left(x_{1}, x_{2}, x_{3}\right):=\triangle\left(\bar{x}_{1}, \bar{x}_{2}, \bar{x}_{3}\right)$ in the Euclidean space $\mathbb{R}^{2}$ such that

$$
d\left(x_{i}, x_{j}\right)=d_{\mathbb{R}^{2}}\left(\bar{x}_{i}, \bar{y}_{j}\right), \forall i, j=1,2,3 .
$$

A geodesic space $X$ is a $\mathrm{CAT}(0)$ space, if for each geodesic triangle $\triangle\left(x_{1}, x_{2}, x_{3}\right)$ in $X$ and its comparison triangle $\bar{\triangle}:=\triangle\left(\bar{x}_{1}, \bar{x}_{2}, \bar{x}_{3}\right)$ in $\mathbb{R}^{2}$, the following comparison axiom called $\mathrm{CAT}(0)$ inequality

$$
d(x, y) \leq d_{\mathbb{R}^{2}}(\bar{x}, \bar{y})
$$

is satisfied for all $x, y \in \triangle$ and all comparison points $\bar{x}, \bar{y} \in \bar{\triangle}$.

The reader interested in detailed study of such spaces is referred to [7] and references therein.

Let $x, y \in X$ and $\lambda \in[0,1]$. Throughout this paper, we will use the notation $\lambda x \oplus(1-\lambda) y$ for the unique point $z \in[x, y]$ satisfying

$$
d(z, x)=(1-\lambda) d(x, y) \quad \text { and } \quad d(z, y)=\lambda d(x, y)
$$

A subset $C$ of a $\mathrm{CAT}(0)$ space is convex if it includes each geodesic segment joining any two points in $C$, that is, $[x, y] \subseteq C$ for all $x, y \in C$.

Berg and Nikolaev [6] introduced the concept of quasilinearization in a metric space $X$ as follows:

Denote a pair $(a, b) \in X \times X$ by $\overrightarrow{a b}$ and call it a vector. The quasilinearization is a map $\langle.,\rangle:.(X \times X) \times(X \times X) \rightarrow \mathbb{R}$ defined by

$$
\langle\overrightarrow{a b}, \overrightarrow{c d}\rangle=\frac{1}{2}\left(d^{2}(a, d)+d^{2}(b, c)-d^{2}(a, c)-d^{2}(b, d)\right), \quad \forall a, b, c, d \in X .
$$

It is easily seen that $\langle\overrightarrow{a b}, \overrightarrow{c d}\rangle=\langle\overrightarrow{c d}, \overrightarrow{a b}\rangle,\langle\overrightarrow{a b}, \overrightarrow{c d}\rangle=-\langle\overrightarrow{b a}, \overrightarrow{c d}\rangle$ and

$$
\langle\overrightarrow{a x}, \overrightarrow{c d}\rangle+\langle\overrightarrow{x b}, \overrightarrow{c d}\rangle=\langle\overrightarrow{a b}, \overrightarrow{c d}\rangle \text { for all } a, b, c, d, x \in X
$$

We say that $X$ satisfies the Cauchy-Schwarz inequality if

$$
\langle\overrightarrow{a b}, \overrightarrow{c d}\rangle \leq d(a, b) d(c, d)
$$

for all $a, b, c, d \in X$. It is known that a geodesically connected metric space is a $\mathrm{CAT}(0)$ space if and only if it satisfies the Cauchy-Schwarz inequality [6].

Using the concept of a quasilinearization, Liu and Chang [34] defined demicontractive mappings in $\mathrm{CAT}(0)$ spaces as follows: 
Definition 2.1. Let $(X, d)$ be a CAT(0) space. A mapping $T: X \rightarrow X$ is said to be demicontractive if $F(T) \neq \emptyset$ and there exists a constant $\lambda \in[0,1)$ such that

$$
\langle\overrightarrow{T x p}, \overrightarrow{x p}\rangle \leq d^{2}(x, p)-\lambda d^{2}(x, T x), \forall x \in X, p \in F(T)
$$

It is easy to show that the above inequality is equivalent to

$$
d^{2}(T x, p) \leq d^{2}(x, p)+(1-2 \lambda) d^{2}(x, T x) .
$$

which implies

$$
d^{2}(T x, p) \leq d^{2}(x, p)+k d^{2}(x, T x), \quad \text { where } k=1-2 \lambda .
$$

Lemma 2.2. [20] [17] Let $X$ be a CAT(0) space, $x, y, z \in X$ and $\lambda \in[0,1]$. Then

(i): $d(\lambda x \oplus(1-\lambda) y, z) \leq \lambda d(x, z)+(1-\lambda) d(y, z)$.

(ii): $d^{2}(\lambda x \oplus(1-\lambda) y, z) \leq \lambda d^{2}(x, z)+(1-\lambda) d^{2}(y, z)-\lambda(1-\lambda) d^{2}(x, y)$.

(iii): $d^{2}(\lambda x \oplus(1-\lambda) y, z) \leq \lambda^{2} d^{2}(x, z)+(1-\lambda)^{2} d^{2}(y, z)+2 \lambda(1-\lambda)\langle\overrightarrow{x z}, \overrightarrow{y z}\rangle$.

Let $\left\{x_{n}\right\}$ be a bounded sequence in a complete $\operatorname{CAT}(0)$ space $X$. For $x \in X$, we set

$$
r\left(x,\left\{x_{n}\right\}\right)=\limsup _{n \rightarrow \infty} d\left(x, x_{n}\right) .
$$

The asymptotic radius $r\left(\left\{x_{n}\right\}\right)$ of $\left\{x_{n}\right\}$ is given by

$$
r\left(\left\{x_{n}\right\}\right)=\inf \left\{r\left(x,\left\{x_{n}\right\}\right): x \in X\right\},
$$

and the asymptotic center $A\left(\left\{x_{n}\right\}\right)$ of $\left\{x_{n}\right\}$ is the set

$$
A\left(\left\{x_{n}\right\}\right)=\left\{x \in X: r\left(x,\left\{x_{n}\right\}\right)=r\left(\left\{x_{n}\right\}\right)\right\} .
$$

It is well known that in a CAT(0) space, $A\left(\left\{x_{n}\right\}\right)$ consists of exactly one point( [19]). A sequence $\left\{x_{n}\right\}$ in $X$ is called $\triangle$-convergent to $x \in X$, denoted by $\triangle-\lim _{n} x_{n}$ $=x$ if $x$ is the unique asymptotic center of $\left\{u_{n}\right\}$, for every subsequence $\left\{u_{n}^{n}\right\}$ of $\left\{x_{n}\right\}$.

Lemma 2.3. [31] Every bounded sequence in a complete CAT(0) space always has a $\triangle$-convergent subsequence.

Lemma 2.4. [18] If $\left\{x_{n}\right\}$ is a bounded sequence in a closed and convex subset $C$ of a complete $C A T(0)$ space, then the asymptotic center of $\left\{x_{n}\right\}$ is in $C$.

Let $\left\{x_{n}\right\}$ be a bounded sequence in a complete $\operatorname{CAT}(0)$ space $X$, and $C$ be a closed and convex subset of $X$ which contains $\left\{x_{n}\right\}$. We employ the notation

$$
\left\{x_{n}\right\} \rightarrow w \Leftrightarrow \limsup _{n \rightarrow \infty} d\left(x_{n}, w\right)=\inf _{x \in C}\left(\limsup _{n \rightarrow \infty} d\left(x_{n}, x\right)\right) .
$$

We note that $\left\{x_{n}\right\} \rightarrow w$ if and only if $A\left(\left\{x_{n}\right\}\right)=\{w\}$ (see [40]).

Lemma 2.5. [40] If $\left\{x_{n}\right\}$ is a bounded sequence in a closed and convex subset $C$ of a complete $C A T(0)$ space, then $\triangle-\lim _{n \rightarrow \infty} x_{n}=p$ implies that $\left\{x_{n}\right\} \rightarrow p$.

Lemma 2.6. [26] Let $\left\{x_{n}\right\}$ be a sequence in a complete $C A T(0)$ space $X$, and $x \in X$. Then $\left\{x_{n}\right\}$ is $\triangle$-convergent to $x$ if and only if $\lim _{\sup _{n \rightarrow \infty}}\left\langle\overrightarrow{x x_{n}}, \overrightarrow{x y}\right\rangle \leq 0$ for all $y \in X$. 
A function $f: C \rightarrow(-\infty, \infty]$ defined on a convex subset $C$ of a CAT(0) space is convex if, for any $x$ and $y$ in $C$ with geodesic segment $[x, y]:=\left\{\gamma_{x, y}(\lambda): 0 \leq \lambda \leq\right.$ $1\}:=\{\lambda x \oplus(1-\lambda) y: 0 \leq \lambda \leq 1\}$, the function $f \circ \gamma$ is convex, that is,

$$
f\left(\gamma_{x, y}(\lambda)\right):=f(\lambda x \oplus(1-\lambda) y) \leq \lambda f(x)+(1-\lambda) f(y) .
$$

For examples of convex functions in $\operatorname{CAT}(0)$, we refer to [12].

For any $\lambda>0$, define the Moreau-Yosida resolvent of $f$ in $\operatorname{CAT}(0)$ space $X$ as follows:

$$
J_{\lambda}(x)=\underset{y \in X}{\arg \min }\left[f(y)+\frac{1}{2 \lambda} d^{2}(y, x)\right], \forall x \in X .
$$

Let $f: X \rightarrow(-\infty, \infty]$ be a proper convex and lower semi-continuous function. It is known [1] that the set $F\left(J_{\lambda}\right)$ of fixed points of the resolvent associated with $f$ coincides with $\arg \min _{y \in X} f(y)$, the set of minimizers of $f$. Also for any $\lambda>0$, the resolvent $J_{\lambda}$ of $f$ is nonexpansive [25].

Lemma 2.7. (Sub-differential inequality [2]) Let $(X, d)$ be a complete $C A T(0)$ space and $f: X \rightarrow(-\infty, \infty]$ a proper convex and lower semi-continuous. Then, for all $x, y \in X$ and $\lambda>0$, the following inequality holds:

$$
\frac{1}{2 \lambda} d^{2}\left(J_{\lambda} x, y\right)-\frac{1}{2 \lambda} d^{2}(x, y)+\frac{1}{2 \lambda} d^{2}\left(x, J_{\lambda} x\right)+f\left(J_{\lambda} x\right) \leq f(y) .
$$

Lemma 2.8. (The resolvent identity $[25,39]$ ) Let $(X, d)$ be a complete $C A T(0)$ space and $f: X \rightarrow(-\infty, \infty]$ a proper convex and lower semi-continuous. Then the following identity holds:

$$
J_{\lambda} x=\left(\frac{\lambda-\mu}{\lambda} J_{\lambda} x \oplus \frac{\mu}{\lambda} x\right)
$$

for all $x \in X$ and $\lambda>\mu>0$.

Lemma 2.9. ([35]) If $\left\{a_{n}\right\}$ is a sequence of real numbers and there exists a subsequence $\left\{n_{i}\right\}$ of $\{n\}$ such that $a_{n_{i}}<a_{n_{i}+1}$ for all $i \in \mathbb{N}$, then there exists a nondecreasing sequence $\left\{m_{k}\right\} \subset \mathbb{N}$ such that $m_{k} \rightarrow \infty$ and the following properties are satisfied:

$$
a_{m_{k}} \leq a_{m_{k}+1} \text { and } a_{k} \leq a_{m_{k}+1} .
$$

for all sufficiently large numbers $k \in \mathbb{N}$. In fact, $m_{k}=\max \left\{j \leq k: a_{j}<a_{j+1}\right\}$.

Lemma 2.10. ( [50]) If $\left\{a_{n}\right\}$ is a sequence of nonnegative real numbers satisfying the following inequality:

$$
a_{n+1} \leq\left(1-\alpha_{n}\right) a_{n}+\alpha_{n} \sigma_{n}+\gamma_{n}, n \geq 0,
$$

where, $(i)\left\{\alpha_{n}\right\} \subset[0,1], \sum \alpha_{n}=\infty$; (ii) $\lim \sup \sigma_{n} \leq 0 ;($ iii $) \gamma_{n} \geq 0 ;(n \geq 0)$ and $\sum \gamma_{n}<\infty$. Then, $a_{n} \rightarrow 0$ as $n \rightarrow \infty$.

The condition of demicontractivity or the more restrictive condition of quasinonexpansivity is not sufficient for the convergence of the Mann iteration, even in finite dimensional spaces; some additional smoothness properties of the mapping $T$ , like the continuity or demiclosedness are required (cf. [14]).

Definition 2.11. [14] A self mapping $T$ on a Banach space is said to be demiclosed at $y$, if for any sequence $\left\{x_{n}\right\}$ which converges weakly to $x$, and if the sequence $\left\{T\left(x_{n}\right)\right\}$ converges strongly to $y$, then $T(x)=y$. 
In particular, if $y=0$, then $T$ is demiclosed at 0 . As the concept of $\Delta$ convergence in $\mathrm{CAT}(0)$ space is analogue of weak convergence in Banach space, so the following is an analogue of Definition 2.11 in $\mathrm{CAT}(0)$ space.

Lemma 2.12. [40] Let $X$ be a complete CAT(0) space. A mapping $T: X \rightarrow X$ is said to be $\Delta$-demiclosed at 0 , if for any bounded sequence $\left\{x_{n}\right\}$ in $X$ such that $\triangle-\lim _{n \rightarrow \infty} x_{n}=p$ and $\lim _{n \rightarrow \infty} d\left(x_{n}, T x_{n}\right)=0$, we have $T p=p$.

\section{MAin RESUlt}

Theorem 3.1. Let $(X, d)$ be a complete CAT(0) space, $f: X \rightarrow(-\infty, \infty]$ a proper convex and lower semi-continuous function and $T: X \rightarrow X$ an L-Lipschitizan demicontractive mapping such that $T$ is $\Delta$-demiclosed at 0 and $\Omega:=F(T) \bigcap \underset{y \in X}{\arg \min } f(y) \neq$ $\emptyset$. If $\left\{\alpha_{n}\right\}_{n=1}^{\infty}$ and $\left\{\beta_{n}\right\}_{n=1}^{\infty}$ are sequences in $(0,1)$ satisfying the following conditions:

(c1) $\lim _{n \rightarrow \infty} \alpha_{n}=0$;

(c2) $\sum_{n=1}^{\infty} \alpha_{n}=\infty$;

(c3) $0<\epsilon \leq \beta_{n}<1-2 k, \forall n \geq 1$ and some $\epsilon>0$ and $k \in[0,1)$,

then the following hybrid proximal point algorithm $\left\{x_{n}\right\}_{n=1}^{\infty}$ defined for any $x_{1} \in$ $X$,

$$
\left\{\begin{array}{l}
z_{n}=\underset{y \in X}{\arg \min }\left[f(y)+\frac{1}{2 \lambda_{n}} d^{2}\left(y, x_{n}\right)\right] \\
y_{n}=\left(\left(1-\alpha_{n}\right) z_{n}\right) \\
x_{n+1}=\left(1-\beta_{n}\right) z_{n} \oplus \beta_{n} T y_{n}
\end{array}\right.
$$

converges strongly to $p \in \Omega$.

Proof. Let $p \in \Omega$. Then $f(p) \leq f(y), \forall y \in X$. Thus, by Lemma 2.7, we obtain

$$
f(p)+\frac{1}{2 \lambda_{n}} d^{2}(p, p) \leq f(y)+\frac{1}{2 \lambda_{n}} d^{2}(y, p), \forall y \in X,
$$

and hence it follows from (2.5) that $p=J_{\lambda_{n}} p, \forall n \geq 1$. Indeed, from (2.4) and (3.1), we have $z_{n}=J_{\lambda_{n}} x_{n}$ and as $J_{\lambda_{n}}$ is nonexpansive (see [25]), so we obtain

$$
d\left(z_{n}, p\right)=d\left(J_{\lambda_{n}} x_{n}, J_{\lambda_{n}} p\right) \leq d\left(x_{n}, p\right) .
$$

Using (3.1) and Lemma 2.2 (ii), we obtain

$$
\begin{aligned}
d^{2}\left(y_{n}, p\right) & \left.=d^{2}\left(\left(1-\alpha_{n}\right) z_{n}\right), p\right) \\
& \leq \alpha_{n} d^{2}(0, p)+\left(1-\alpha_{n}\right) d^{2}\left(z_{n}, p\right)-\alpha_{n}\left(1-\alpha_{n}\right) d^{2}\left(0, z_{n}\right) .
\end{aligned}
$$


Also from (3.1) and (2.3), we obtain

$$
\begin{aligned}
d^{2}\left(x_{n+1}, p\right)= & d^{2}\left(\left(1-\beta_{n}\right) z_{n} \oplus \beta_{n} T y_{n}, p\right) \\
\leq & \left(1-\beta_{n}\right) d^{2}\left(z_{n}, p\right)+\beta_{n} d^{2}\left(T y_{n}, p\right)-\beta_{n}\left(1-\beta_{n}\right) d^{2}\left(z_{n}, T y_{n}\right) \\
\leq & \left(1-\beta_{n}\right) d^{2}\left(z_{n}, p\right)+\beta_{n}\left[d^{2}\left(y_{n}, p\right)+k d^{2}\left(y_{n}, T y_{n}\right)\right] \\
& -\beta_{n}\left(1-\beta_{n}\right) d^{2}\left(z_{n}, T y_{n}\right) \\
\leq & \left(1-\beta_{n}\right) d^{2}\left(z_{n}, p\right)+\beta_{n} d^{2}\left(y_{n}, p\right)+\beta_{n} k\left[d\left(y_{n}, z_{n}\right)+\left(z_{n}, T y_{n}\right)\right]^{2} \\
& -\beta_{n}\left(1-\beta_{n}\right) d^{2}\left(z_{n}, T y_{n}\right) \\
\leq & \left(1-\beta_{n}\right) d^{2}\left(z_{n}, p\right)+\beta_{n} d^{2}\left(y_{n}, p\right)+2 k \beta_{n} d^{2}\left(y_{n}, z_{n}\right) \\
& -\beta_{n}\left(1-\beta_{n}-2 k\right) d^{2}\left(z_{n}, T y_{n}\right) \\
\leq & \left(1-\beta_{n}\right) d^{2}\left(z_{n}, p\right)+\beta_{n} d^{2}\left(y_{n}, p\right) \\
& +\beta\left[\alpha_{n} d^{2}\left(0, z_{n}\right)+\left(1-\alpha_{n}\right) d^{2}\left(z_{n}, z_{n}\right)-\alpha_{n}\left(1-\alpha_{n}\right) d^{2}\left(0, z_{n}\right)\right] \\
& -\beta_{n}\left(1-\beta_{n}-2 k\right) d^{2}\left(z_{n}, T y_{n}\right) \\
\leq & \left(1-\alpha_{n}\right) d^{2}\left(z_{n}, p\right)+\beta_{n} d^{2}\left(y_{n}, p\right)+2 k \beta_{n} \alpha_{n}^{2} d^{2}\left(0, z_{n}\right) \\
& -\beta_{n}\left(1-\beta_{n}-2 k\right) d^{2}\left(z_{n}, T y_{n}\right) \\
\leq & \left(1-\beta_{n}\right) d^{2}\left(z_{n}, p\right)+\alpha_{n} \beta_{n} d^{2}(0, p)+\left(1-\alpha_{n}\right) \beta_{n} d^{2}\left(z_{n}, p\right) \\
& -\alpha_{n} \beta_{n}\left(1-\alpha_{n}\right) d^{2}\left(0, z_{n}\right)+2 k \beta_{n} \alpha_{n}^{2} d^{2}\left(0, z_{n}\right) \\
& -\beta_{n}\left(1-\beta_{n}-2 k\right) d^{2}\left(z_{n}, T y_{n}\right) \\
\leq & \left(1-\alpha_{n} \beta_{n}\right) d^{2}\left(x_{n}, p\right)+\left(1-\alpha_{n} \beta_{n}\right) d^{2}(0, p) \\
& -\alpha_{n} \beta_{n}\left[1-\alpha_{n}-2 k \alpha_{n}\right] d^{2}\left(0, z_{n}\right) \\
& -\beta_{n}\left(1-\beta_{n}-2 k\right) d^{2}\left(z_{n}, T y_{n}\right) \\
\leq & \left(1-\alpha_{n} \beta_{n}\right) d^{2}\left(x_{n}, p\right)+\left(1-\alpha_{n} \beta_{n}\right) d^{2}(0, p) \\
\leq & \max \left\{d^{2}\left(x_{n}, p\right), d^{2}(0, p)\right\} . \\
&
\end{aligned}
$$

By induction, we obtain

$$
d^{2}\left(x_{n}, p\right) \leq \max \left\{d^{2}\left(x_{1}, p\right), d^{2}(0, p)\right\} .
$$

Thus $\left\{x_{n}\right\}_{n=1}^{\infty}$ is bounded, and hence $\left\{y_{n}\right\}_{n=1}^{\infty}$ is bounded. By (3.1) and Lemma 2.2 (iii), we have

$$
d^{2}\left(y_{n}, p\right) \leq \alpha_{n}^{2} d^{2}(0, p)+\left(1-\alpha_{n}\right)^{2} d^{2}\left(z_{n}, p\right)+2 \alpha_{n}\left(1-\alpha_{n}\right)\left\langle\overrightarrow{0 p}, \overrightarrow{z_{n} p}\right\rangle .
$$

Substituting (3.3) into (3.2), we obtain

$$
\begin{aligned}
d^{2}\left(x_{n+1}, p\right) \leq & \left(1-\alpha_{n} \beta_{n}\right) d^{2}\left(z_{n}, p\right)+2 \alpha_{n} \beta_{n}\left[\alpha_{n} d^{2}(0, p)+2 k \alpha_{n} d^{2}\left(0, z_{n}\right)\right. \\
& \left.+2\left(1-\alpha_{n}\right)\left\langle\overrightarrow{0 p}, \overrightarrow{z_{n} p}\right\rangle\right]-\beta_{n}\left(1-\beta_{n}-2 k\right) d^{2}\left(z_{n}, T y_{n}\right) \\
\leq & \left(1-\alpha_{n} \beta_{n}\right) d^{2}\left(x_{n}, p\right)+2 \alpha_{n} \beta_{n}\left[\alpha_{n} d^{2}(0, p)+2 k \alpha_{n} d^{2}\left(0, z_{n}\right)\right. \\
& \left.+2\left(1-\alpha_{n}\right)\left\langle\overrightarrow{0 p}, \overrightarrow{z_{n} p}\right\rangle\right]-\beta_{n}\left(1-\beta_{n}-2 k\right) d^{2}\left(z_{n}, T y_{n}\right) \\
\leq & \left(1-\alpha_{n} \beta_{n}\right) d^{2}\left(x_{n}, p\right)+2 \alpha_{n} \beta_{n}\left[\alpha_{n} d^{2}(0, p)\right. \\
& \left.+2 k \alpha_{n} d^{2}\left(0, z_{n}\right)+2\left(1-\alpha_{n}\right)\left\langle\overrightarrow{0 p}, \overrightarrow{z_{n} p}\right\rangle\right] .
\end{aligned}
$$

As $\left\{x_{n}\right\}$ and $\left\{z_{n}\right\}$ are bounded, so there exists $D>0$ such that

$$
\left[\alpha_{n} d^{2}(0, p)+2 k \alpha_{n} d^{2}\left(0, z_{n}\right)+2\left(1-\alpha_{n}\right)\left\langle\overrightarrow{0 p}, \overrightarrow{x_{n} p}\right\rangle\right] \leq D .
$$


Now from (3.4), we obtain

$$
\begin{aligned}
& \beta_{n}\left(1-\beta_{n}-2 k\right) d^{2}\left(z_{n}, T y_{n}\right) \leq d^{2}\left(x_{n}, p\right)-d^{2}\left(x_{n+1}, p\right) \\
& \quad+\alpha_{n} \beta_{n}\left[D-d^{2}\left(x_{n}, p\right)\right] .
\end{aligned}
$$

We have now the following two cases.

Case 1: Assume that $\left\{d\left(x_{n}, p\right)\right\}$ is non-increasing. Then $\left\{d\left(x_{n}, p\right)\right\}$ is convergent. It follows from (3.6) that

$$
\beta_{n}\left(1-\beta_{n}-2 k\right) d^{2}\left(z_{n}, T y_{n}\right) \rightarrow 0 \text { as } n \rightarrow \infty
$$

which implies that

$$
d\left(z_{n}, T y_{n}\right) \rightarrow 0 \text { as } n \rightarrow \infty
$$

Now from (3.1) and Lemma 2.2 (i), we obtain

$$
\begin{aligned}
d\left(y_{n}, z_{n}\right) & =d\left(\left(\left(1-\alpha_{n}\right) z_{n}\right), z_{n}\right) \\
& \leq \alpha_{n} d\left(0, z_{n}\right)+\left(1-\alpha_{n}\right) d\left(z_{n}, z_{n}\right) \rightarrow 0 \text { as } n \rightarrow \infty .
\end{aligned}
$$

By (3.7) and (3.8), we obtain that

$$
d\left(y_{n}, T y_{n}\right) \leq d\left(y_{n}, z_{n}\right)+d\left(z_{n}, T y_{n}\right) \rightarrow 0 \text { as } n \rightarrow \infty .
$$

Note that

$$
\frac{1}{2 \lambda_{n}} d^{2}\left(z_{n}, p\right)-\frac{1}{2 \lambda_{n}} d^{2}\left(x_{n}, p\right)+\frac{1}{2 \lambda_{n}} d^{2}\left(x_{n}, z_{n}\right) \leq f(p)-f\left(z_{n}\right) .
$$

As $f(p) \leq f\left(z_{n}\right)$ for all $n \geq 1$, so we have

$$
d^{2}\left(x_{n}, z_{n}\right) \leq d^{2}\left(x_{n}, p\right)-d^{2}\left(z_{n}, p\right) .
$$

Thus

$$
\begin{aligned}
d^{2}\left(x_{n+1}, p\right) \leq & \left(1-\beta_{n}\right) d^{2}\left(z_{n}, p\right)+\beta_{n} d^{2}\left(y_{n}, p\right) 2 k \alpha_{n}^{2} \beta_{n} d^{2}\left(0, z_{n}\right) \\
\leq & \left(1-\beta_{n}\right) d^{2}\left(x_{n}, p\right)+\alpha_{n} \beta_{n} d^{2}(0, p)+\left(1-\alpha_{n}\right) \beta_{n} d^{2}\left(z_{n}, p\right) \\
& -\alpha_{n} \beta_{n}\left(1-\alpha_{n}-2 k \alpha_{n}\right) d^{2}\left(0, z_{n}\right) \\
\leq & \left(1-\beta_{n}\right) d^{2}\left(x_{n}, p\right)+\alpha_{n} \beta_{n} d^{2}(0, p)+\left(1-\alpha_{n}\right) \beta_{n} d^{2}\left(z_{n}, p\right) .
\end{aligned}
$$

Hence

$$
\begin{aligned}
d^{2}\left(x_{n}, p\right) \leq & \frac{1}{\beta_{n}}\left(d^{2}\left(x_{n}, p\right)-d^{2}\left(x_{n+1}, p\right)\right) \\
& +\alpha_{n} d^{2}(0, p)+\left(1-\alpha_{n}\right) d^{2}\left(z_{n}, p\right) .
\end{aligned}
$$

Now by (3.10) and (3.11), we have

$$
\begin{aligned}
d^{2}\left(x_{n}, z_{n}\right) \leq & \frac{1}{\beta_{n}}\left(d^{2}\left(x_{n}, p\right)-d^{2}\left(x_{n+1}, p\right)\right) \\
& +\alpha_{n}\left(d^{2}(0, p)-d^{2}\left(z_{n}, p\right)\right) .
\end{aligned}
$$

Since $\left\{x_{n}\right\}$ and $\left\{z_{n}\right\}$ are bounded and $\left\{d\left(x_{n}, p\right)\right\}$ is non-increasing sequence, therefore $\lim _{n \rightarrow \infty} d\left(x_{n}, z_{n}\right)=0$ and $\lim _{n \rightarrow \infty} d\left(x_{n}, y_{n}\right)=0$. Also from (3.9), we obtain that

$$
\lim _{n \rightarrow \infty} d\left(x_{n}, T y_{n}\right)=0
$$

and

$$
\begin{aligned}
d\left(x_{n}, T x_{n}\right) & \leq d\left(x_{n}, y_{n}\right)+d\left(y_{n}, T y_{n}\right)+d\left(T y_{n}, T x_{n}\right) \\
& \leq(1+L) d\left(x_{n}, y_{n}\right)+d\left(y_{n}, T y_{n}\right) .
\end{aligned}
$$


Thus

$$
\lim _{n \rightarrow \infty} d\left(x_{n}, T x_{n}\right)=0 .
$$

As $\lambda_{n} \geq \lambda>0$, so by Lemma 2.8 and Lemma 2.2(i), we obtain

$$
\begin{aligned}
d\left(J_{\lambda} x_{n}, J_{\lambda_{n}} x_{n}\right) & =d\left(J_{\lambda} x_{n}, J_{\lambda}\left(\frac{\lambda_{n}-\lambda}{\lambda_{n}} J_{\lambda_{n}} x_{n} \oplus \frac{\lambda}{\lambda_{n}} x_{n}\right)\right) \\
& \leq d\left(x_{n},\left(1-\frac{\lambda}{\lambda_{n}}\right) J_{\lambda_{n}} x_{n} \oplus \frac{\lambda}{\lambda_{n}} x_{n}\right) \\
& \leq\left(1-\frac{\lambda}{\lambda_{n}}\right) d\left(x_{n}, z_{n}\right) \rightarrow 0 \text { as } n \rightarrow \infty .
\end{aligned}
$$

Also,

$$
d\left(x_{n}, J_{\lambda} x_{n}\right) \leq d\left(x_{n}, z_{n}\right)+d\left(z_{n}, J_{\lambda} x_{n}\right) \rightarrow 0 \text { as } n \rightarrow \infty .
$$

Since $\left\{x_{n}\right\}$ is bounded and $X$ is a complete CAT(0) space, therefore by Lemma 2.3 , there exists a subsequence $\left\{x_{n_{i}}\right\}$ of $\left\{x_{n}\right\}$ such that $\triangle-\lim x_{n_{i}}=v \in X$. Then, from (3.12) and the fact that $T$ is $\Delta$-demiclosed at 0 , we obtain that $v \in F(T)$. From (3.13) and the fact that $J_{\lambda}$ is nonexpansive [25], we have $v \in \Omega$. By Lemma 2.6 , we have

$$
\lim \sup \left\langle\overrightarrow{0 v}, \overrightarrow{x_{n} \vec{v}}\right\rangle \leq 0
$$

Note that from (2.1) and (2.2), we obtain

$$
\begin{aligned}
\left\langle\overrightarrow{0 v}, \overrightarrow{z_{n} v}\right\rangle & =\left\langle\overrightarrow{0 v}, \overrightarrow{z_{n} x_{n}}\right\rangle+\left\langle\overrightarrow{0 v}, \overrightarrow{x_{n} \vec{v}}\right\rangle \\
& \leq d(0, v) d\left(z_{n}, x_{n}\right)+\left\langle\overrightarrow{0 v}, \overrightarrow{x_{n} v}\right\rangle
\end{aligned}
$$

so from $\lim _{n \rightarrow \infty} d\left(x_{n}, z_{n}\right)=0$ and (3.14), we have

$$
\lim \sup \left\langle\overrightarrow{0 v}, \overrightarrow{z_{n}} \vec{v}\right\rangle \leq 0
$$

Using $v:=p$ in (3.5), we obtain that

$$
d^{2}\left(x_{n+1}, p\right) \leq\left(1-\alpha_{n} \beta_{n}\right) d^{2}\left(x_{n}, v\right)+2 \alpha_{n} \beta_{n}\left[\alpha_{n} d^{2}(0, v)+2 k \alpha_{n} d^{2}\left(0, z_{n}\right)\right.
$$

Consequently,

$$
\left.\left.+\left(1-\alpha_{n}\right)\left\langle\overrightarrow{0 v}, \overrightarrow{z_{n}}\right\rangle\right\rangle\right]
$$

$$
d^{2}\left(x_{n+1}, v\right) \leq\left(1-\alpha_{n} \beta_{n}\right) d^{2}\left(x_{n}, v\right)+\alpha_{n} \beta_{n} \sigma_{n},
$$

where $\sigma_{n}:=\alpha_{n} d^{2}(0, v)+2 k \alpha_{n} d^{2}\left(0, z_{n}\right)+\left(1-\alpha_{n}\right)\left\langle\overrightarrow{0 v}, \overrightarrow{z_{n} v}\right\rangle$. It follows from Lemma 2.10 that $d\left(x_{n}, v\right) \rightarrow 0$ as $n \rightarrow \infty$, that is, $x_{n} \rightarrow v$ as $n \rightarrow \infty$.

Case 2: Assume that $\left\{d\left(x_{n}, p\right)\right\}_{n \geq 1}$ is non-decreasing sequence. Now, there exists a subsequence $\left\{n_{i}\right\}$ of $\{n\}$ such that

$$
d\left(x_{n_{i}}, p\right)<d\left(x_{n_{i}+1}, p\right)
$$

for all $i \in \mathbb{N}$. Now by Lemma 2.9, there exists an increasing sequence $\left\{m_{j}\right\}_{j \geq 1}$ such that $m_{j} \rightarrow \infty, d\left(x_{m_{j}}, p\right) \leq d\left(x_{m_{j}+1}, p\right)$ and $d\left(x_{j}, p\right) \leq d\left(x_{m_{j}+1}, p\right)$ for all $j \geq 1$. Also from (3.6) and the fact that $\alpha_{m_{j}} \rightarrow 0$, we obtain

$$
\begin{aligned}
\beta_{m_{j}}\left(1-\beta_{m_{j}}-2 k\right) d^{2}\left(z_{m_{j}}, T x_{m_{j}}\right) \leq & d^{2}\left(x_{m_{j}}, p\right)-d^{2}\left(x_{m_{j}+1}, p\right) \\
& +\alpha_{m_{j}} \beta_{m_{j}}\left[D-d^{2}\left(x_{m_{j}}, p\right)\right] .
\end{aligned}
$$

This implies $d\left(z_{m_{j}}, T x_{m_{j}}\right) \rightarrow 0$ as $j \rightarrow \infty$. Thus, as in Case 1 , we obtain that $d\left(x_{m_{j}}, T x_{m_{j}}\right) \rightarrow 0$ as $j \rightarrow \infty$. Following arguments similar to those in the proof of 
Case 1, we get $\lim \sup \left\langle\overrightarrow{0 v}, \overrightarrow{x_{m_{j}}} \vec{v}\right\rangle \leq 0$. Also from the inequality (3.15), we obtain that,

$$
\begin{aligned}
d^{2}\left(x_{m_{j}+1}, p\right) \leq & \left(1-\alpha_{m_{j}} \beta_{m_{j}}\right) d^{2}\left(x_{m_{j}}, v\right) \\
& +2 \alpha_{m_{j}} \beta_{m_{j}}\left[\alpha_{m_{j}} d^{2}(0, v)+2 k \alpha_{m_{j}} d^{2}\left(0, z_{m_{j}}\right)\right. \\
& \left.+\left(1-\alpha_{m_{j}}\right)\left\langle\overrightarrow{0 v}, \overrightarrow{z_{m_{j}}}\right\rangle\right]
\end{aligned}
$$

which implies that

$$
\begin{aligned}
\alpha_{m_{j}} \beta_{m_{j}} d^{2}\left(x_{m_{j}}, p\right) \leq & d^{2}\left(x_{m_{j}}, v\right)-d\left(x_{m_{j}+1}, p\right)+2 \alpha_{m_{j}} \beta_{m_{j}}\left[\alpha_{m_{j}} d^{2}(0, v)\right. \\
& \left.+2 k \alpha_{m_{j}} d^{2}\left(0, z_{m_{j}}\right)+\left(1-\alpha_{m_{j}}\right)\left\langle\overrightarrow{0 v}, \overrightarrow{z_{m_{j}}} \overrightarrow{ }\right\rangle\right]
\end{aligned}
$$

In particular, $d^{2}\left(x_{m_{j}}, v\right) \leq d^{2}\left(x_{m_{j}+1}, v\right)$ and $\alpha_{m_{j}} \beta_{m_{j}}>0$ give that

$$
d^{2}\left(v, x_{m_{j}}\right) \leq\left[\alpha_{m_{j}} d^{2}(0, v)+2 k \alpha_{m_{j}} d^{2}\left(0, z_{m_{j}}\right)+\left(1-\alpha_{m_{j}}\right)\left\langle\overrightarrow{0 v}, \overrightarrow{z_{m_{j}}}\right\rangle\right] .
$$

Using limsup $\left\langle\overrightarrow{0 v}, \overrightarrow{z_{m_{j}}} \vec{v}\right\rangle \leq 0$ and the fact that $\alpha_{m_{j}} \rightarrow 0$ as $j \rightarrow \infty$, we obtain that $d\left(x_{m_{j}}, v\right) \rightarrow 0$ as $j \rightarrow \infty$. This together with (3.16) implies that $d\left(x_{m_{j}+1}, v\right) \rightarrow 0$ as $j \rightarrow \infty$. But $d\left(x_{j}, v\right) \leq d\left(x_{m_{j}+1}, v\right)$, for all $j \geq 1$. Thus we obtain that $x_{j} \rightarrow v$. Hence the result follows.

Remark 3.2. Theorem 3.1 extends the result of Bačák [4] from weak convergence to strong convergence and the result of Cholamjiak et al. [15] from nonexpanvive mapping to Lipschitzian demicontractive mapping. Also Theorem 3.1 extended the result in [36] from strict pseudo-contractive mapping in a real Hilbert space to Lipschitzian demicontractive mapping in a more general space than Hilbert space. We studied a new hybrid proximal point algorithm for solving convex minimization problem as well as fixed point problem of Lipschitzian demicontractive mappings in CAT(0) spaces. Our method of proof is different from that of Cholamjiak et al. [15] and Chang et al. [12].

Remark 3.3. Since every Hilbert space is a complete CAT(0) space [28], therefore Theorem 3.1 is an extension and generalization of the results of Guler [24], Rockafellar [45] and Kamimura and Takahashi [48].

\section{Applications}

Let $C$ be a nonempty closed and convex subset of a real Hilbert space. The normal cone for $C$ at a point $x \in C$, denoted by $N_{C}(x)$, is defined by

$$
N_{C}(x)=\left\{x^{*} \in H:\left\langle y-x, x^{*}\right\rangle \leq 0, \forall y \in C\right\} .
$$

For a proper lower semicontinuous convex function $f: H \rightarrow(-\infty, \infty]$, the subdifferential mapping $\partial f$ of $f$ is defined by

$$
\partial f(x)=\left\{x^{*} \in H: f(x)+\left\langle y-x, x^{*}\right\rangle \leq f(y), \forall x, y \in H\right\} .
$$

Rockafellar [46] proved that $\partial f$ in (4.1) is a maximal monotone operator. The mapping $(I+\lambda \partial f)^{-1}$ is called the resolvent of the operator $\partial f$ with parameter $\lambda>0$, so the proximal operator is equivalent to resolvent of the subdifferential 
operator, that is,

$$
\begin{aligned}
x & =\arg \min _{x}\left(f(x)+\frac{1}{2 \lambda}\|x-u\|_{2}^{2}\right) \\
& \Leftrightarrow \quad 0 \in \partial f(x)+\frac{1}{\lambda}(x-u) \\
& \Leftrightarrow \quad u \in x+\lambda \partial f(x) \\
& \Leftrightarrow \quad x \in(I+\lambda \partial f)^{-1}(u) .
\end{aligned}
$$

Let $i_{C}$ be the indicator function of $C$, that is,

$$
i_{C}(x)= \begin{cases}0, & \text { if } x \in C \\ \infty, & \text { if } x \notin C\end{cases}
$$

Then $i_{C}: H \rightarrow(-\infty, \infty]$ is a lower semicontinuous function on $H$ and $\partial i_{C}$ is a maximal monotone mapping. Let $J_{\lambda} x=\left(I+\lambda \partial i_{C}\right)^{-1} x$ for all $\lambda>0$ and $x \in H$. From the fact that $\partial i_{C} x=N_{C} x$ for $x \in C$, (see [47]), we obtain

$$
\begin{aligned}
u \in J_{\lambda} & \Leftrightarrow x \in u+\lambda \partial i_{C} u \Leftrightarrow x \in u+\lambda N_{C} u \\
& \Leftrightarrow x-u \in \lambda N_{C} u \Leftrightarrow\langle x-u, y-u\rangle \leq 0, \forall y \in C \\
& \Leftrightarrow u=P_{C} x ;
\end{aligned}
$$

but $x \in(I+\lambda \partial f)^{-1}(u)$, and so it follows that

$$
\begin{aligned}
x & =\arg \min _{x}\left(i_{C}(x)+\frac{1}{2 \lambda}\|x-u\|_{2}^{2}\right) \\
& \Leftrightarrow x \in\left(A+\partial i_{C}\right)^{-1}(0) \\
& \Leftrightarrow 0 \in\left(A+\partial i_{C}\right) x \Leftrightarrow-A x \in i_{C} x \\
& \Leftrightarrow\langle-A x, y-x\rangle \leq 0, \forall y \in C \\
& \Leftrightarrow x \in V I(C, A)
\end{aligned}
$$

and hence

$$
\begin{aligned}
x & =\arg \min _{x}\left(f(x)+\frac{1}{2 \lambda}\|x-u\|_{2}^{2}\right) \\
& \Leftrightarrow x \in\left(A+\partial i_{C}\right)^{-1}(0) \\
& \Leftrightarrow x \in V I(C, A) .
\end{aligned}
$$

Now using Theorem 3.1, we obtain strong convergence theorems for approximating a fixed point of an $L$-Lipschitizan demicontractive mapping and solution of the variational inequality problem for $\gamma$-inverse monotone mapping.

Theorem 4.1. Let $C$ be a nonempty closed and convex subset of a real Hilbert space $H, T: C \rightarrow C$ an L-Lipschitizan demicontractive mapping such that $T$ is demiclosed at 0 and $A: C \rightarrow H$ be a $\gamma$-inverse strongly monotone mapping and $B: C \rightarrow H$ be a maximal monotone such that $F(T) \cap V I(C, A) \neq \emptyset$. If $\left\{\alpha_{n}\right\}_{n=1}^{\infty}$, $\left\{\beta_{n}\right\}_{n=1}^{\infty}$ are sequences in $(0,1)$ and $\left\{\lambda_{n}\right\} \in(a, b) \subset(a, 2 \lambda)$ satisfying the following conditions:

(c1) $\lim _{n \rightarrow \infty} \alpha_{n}=0$;

(c2) $\sum_{n=1}^{\infty} \alpha_{n}=\infty$;

(c3) $0<\epsilon \leq \beta_{n}<1-2 k$, 
then the following hybrid proximal point algorithm $\left\{x_{n}\right\}_{n=1}^{\infty}$ defined for any $x_{1} \in C$,

$$
\left\{\begin{array}{l}
z_{n}=P_{C}\left(x_{n}-\lambda_{n} A x_{n}\right) \\
y_{n}=P_{C}\left(\left(1-\alpha_{n}\right) z_{n}\right) \\
x_{n+1}=\left(1-\beta_{n}\right) z_{n}+\beta_{n} T y_{n}
\end{array}\right.
$$

converges strongly to the minimum-norm point $p$ of $F(T) \bigcap V I(C, A)$.

Proof. Put $B x=\partial i_{C} x$. The conclusion follows from Theorem 3.1 in view of (4.4).

Theorem 4.2. Let $C$ be a nonempty closed and convex subset of a real Hilbert space $H, T: C \rightarrow C$ an L-Lipschitizan demicontractive mapping such that $T$ is demiclosed at 0 and $A, B: C \rightarrow H$ be maximal monotone mappings such that $\Omega:=F(T) \bigcap(A+B)^{-1}(0) \neq \emptyset$. If $\left\{\alpha_{n}\right\}_{n=1}^{\infty},\left\{\beta_{n}\right\}_{n=1}^{\infty}$ are sequences in $(0,1)$ satisfying the following conditions:

(c1) $\lim _{n \rightarrow \infty} \alpha_{n}=0$;

(c2) $\sum_{n=1}^{\infty} \alpha_{n}=\infty$;

(c3) $0<\epsilon \leq \beta_{n}<1-2 k$, $C$,

then the following hybrid proximal point algorithm $\left\{x_{n}\right\}_{n=1}^{\infty}$ defined for any $x_{1} \in$

$$
\left\{\begin{array}{l}
y_{n}=P_{C}\left(\left(1-\alpha_{n}\right) U_{\lambda_{n}}\left(x_{n}-\lambda_{n} A x_{n}\right)\right) \\
x_{n+1}=\left(1-\beta_{n}\right) U_{\lambda_{n}} x_{n}+\beta_{n} T y_{n}
\end{array}\right.
$$

( here $U_{\lambda_{n}} x_{n}:=\left(I+\lambda_{n} B\right)^{-1} x_{n}$ and $\lambda_{n} \subset(a, 1), a>0$ ) converges strongly to the minimum-norm point $x^{*}$ of $\Omega$.

Proof. We know that $\partial f$ is maximal monotone. From (4.2) and (4.3), we have that $0 \in \partial f(x)$ if and only if $f(x)=\min _{y \in H} f(y)$ and $y \in\left(I+\partial i_{C}\right)^{-1}(x)$ if and only if $y=P_{C} x$ for all $x \in H, y \in C$. The conclusion now follows from Theorem 3.1.

4.1. Numerical example. We give an example in support of Theorem 3.1. Let $X=\mathbb{R}^{4}$ be endowed with Euclidean norm $\|.\|_{2}$. For each $x=\left(x^{(1)}, x^{(2)}, x^{(3)}, x^{(4)}\right) \in$ $X$, let $A: X \rightarrow X$ be defined by $A(x)=\left(2 x^{(1)}+x^{(3)}, x^{(2)}+x^{(4)}, x^{(3)}-x^{(1)}, 2 x^{(4)}-\right.$ $\left.x^{(2)}\right)$, which is continuous linear and monotone mapping. We define $f: X \rightarrow$ $(-\infty, \infty]$ by

where

$$
f(x)=\frac{1}{2}\|A x-a\|_{2}^{2},
$$

$$
A=\left(\begin{array}{cccc}
2 & 0 & 1 & 0 \\
0 & 1 & 0 & 1 \\
-1 & 0 & 1 & 0 \\
0 & -1 & 0 & 2
\end{array}\right) \quad \text { and } \quad a=\left(\begin{array}{l}
0 \\
0 \\
0 \\
0
\end{array}\right)
$$

The function $f$ is proper convex and lower semi continuous function, (cf. [16, 38]). Then, for $\lambda_{n}=1$ for each $n \geq 1$, we obtain from (cf. [38]) that

$$
\begin{aligned}
z_{n}:=\operatorname{Prox}_{f}(x) & =\underset{y \in \mathbb{X}}{\arg \min }\left[f(y)+\frac{1}{2 \lambda_{n}} d^{2}\left(y, x_{n}\right)\right] \\
& =\left(I+A^{T} A\right)^{-1}\left(x+A^{T} a\right) \\
& =\left(I+A^{T} A\right)^{-1} x .
\end{aligned}
$$


Let $x=\left(x^{(1)}, x^{(2)}, x^{(3)}, x^{(4)}\right) \in X$ and $T$ be a self mapping on $X$ defined by $T x=$ $-\frac{3}{2} x$. Then $T$ is a demicontractive mapping with $k=\frac{1}{5}$. Now $\alpha_{n}=\frac{1}{10 n+7}, \beta_{n}=$ $\frac{3 n-1}{50 n}$ satisfy Condition (c1)-(c3) in Theorem 3.1. Therefore, for any $x_{1} \in X$, algorithm (3.1) in view of (4.7) becomes

$$
\left\{\begin{array}{l}
z_{n}=\left(I+A^{T} A\right)^{-1} x_{n} \\
y_{n}=\frac{10 n+6}{10 n+7} z_{n} \\
x_{n+1}=\frac{47 n+1}{50 n} z_{n}+\frac{3-9 n}{100 n} y_{n}, n \geq 1
\end{array}\right.
$$

By (1.3), the algorithm of Cholamjiak et al. [15], using $T_{i} x=\frac{9 x}{10}$ for each $i=1,2$ and $\alpha_{n}=\frac{n}{10 n+1}, \beta_{n}=\frac{3 n-1}{50 n}$, we obtain by (4.7)

$$
\left\{\begin{array}{l}
z_{n}=\left(I+A^{T} A\right)^{-1} x_{n} \\
y_{n}=\frac{9 n+1}{10 n+1} x_{n}+\frac{9 n}{10(10 n+1)} z_{n} \\
x_{n+1}=\frac{9(47 n+1)}{500 n} x_{n}+\frac{27 n-9}{500 n} y_{n}, n \geq 1
\end{array}\right.
$$

By (1.4), the algorithm of Chang et al. [12], using $T_{i} x=S_{i} x=\frac{9 x}{10}$ for each $i=1,2$ and $\alpha_{n}=\frac{n}{10 n+1}, \beta_{n}=\frac{3 n-1}{50 n} \gamma_{n}=1-\left(\alpha_{n}+\beta_{n}\right), \delta_{n}=\frac{2 n+1}{6 n+1}, \eta_{n}=\frac{3 n-2}{6 n+1}, \xi_{n}=\frac{n+2}{6 n+1}$, we obtain by (4.7),

$$
\left\{\begin{array}{l}
z_{n}=\left(I+A^{T} A\right)^{-1} x_{n} \\
y_{n}=\frac{75 n^{2}-9 n-1}{50 n(10 n+1)} x_{n}+\frac{9\left(420 n^{2}+57 n+1\right)}{500 n(10 n+1)} z_{n} \\
x_{n+1}=\frac{45 n-9}{10(6 n+1)} x_{n}+\frac{9 n+18}{10(6 n+1)} y_{n}, n \geq 1
\end{array}\right.
$$

The following tables show results of our numerical experiment based on MATLAB software.

\begin{tabular}{|l|c|c|}
\hline No. of iterations & $x_{n}=\left(x_{n}^{(1)}, x_{n}^{(2)}, x_{n}^{(3)}, x_{n}^{(4)}\right)$ & Erros $=\left\|x_{n}-x_{n-1}\right\|_{2}$ \\
\hline \hline 1 & $(7.0000,6.0000,3.0000,2.0000)$ & \\
2 & $(0.9829,2.7150,0.4275,-0.0088)$ & 7.5928 \\
3 & $(0.1548,1.3126,-0.0659,-0.2420)$ & 1.7176 \\
4 & $(0.0419,0.6624,-0.1170,-0.1845)$ & 0.6644 \\
5 & $(0.0205,0.3425,-0.0867,-0.1126)$ & 0.3300 \\
$\vdots$ & $\vdots$ & $\vdots$ \\
19 & $(0.0396,0.4790,-0.1738,-0.1882) e^{-} 04$ & 0.0000 \\
\hline \hline
\end{tabular}

TABLE 1. Numerical results for the algorithm (4.8)

Acknowledgements: The author A. R. Khan would like to acknowledge the support provided by the Deanship of Scientific Research (DSR) at King Fahd University of Petroleum and Minerals (KFUPM) for funding this work through project No. IN151014.

The authors very much appreciate careful reading of the manuscript by the reviewer and wish to thank him/her for an excellent review and suggested corrections that have helped to improve quality and the presentation of the manuscript. 


\begin{tabular}{|l|c|c|}
\hline No. of iterations & $x_{n}=\left(x_{n}^{(1)}, x_{n}^{(2)}, x_{n}^{(3)}, x_{n}^{(4)}\right)$ & Erros $=\left\|x_{n}-x_{n-1}\right\|_{2}$ \\
\hline \hline 1 & $(7.0000,6.0000,3.0000,2.0000)$ & \\
2 & $(6.2803,5.3892,2.6916,1.7934)$ & 1.0143 \\
3 & $(5.6291,4.8376,2.4125,1.6064)$ & 0.9172 \\
4 & $(5.0437,4.3415,2.1616,1.4382)$ & 0.8246 \\
5 & $(4.5184,3.8958,1.9365,1.2874)$ & 0.7403 \\
$\vdots$ & $\vdots$ & $\vdots$ \\
19 & $(0.9645,0.8528,0.4133,0.2715)$ & 0.1600 \\
\hline \hline
\end{tabular}

TABLE 2. Numerical results for the algorithm (4.9)

\begin{tabular}{|l|c|c|}
\hline No. of iterations & $x_{n}=\left(x_{n}^{(1)}, x_{n}^{(2)}, x_{n}^{(3)}, x_{n}^{(4)}\right)$ & Erros $=\left\|x_{n}-x_{n-1}\right\|_{2}$ \\
\hline \hline 1 & $(7.0000,6.0000,3.0000,2.0000)$ & \\
2 & $(4.2571,4.2742,1.8266,1.1196)$ & 3.5572 \\
3 & $(2.9551,3.2895,1.2580,0.7189)$ & 1.7745 \\
4 & $(2.1430,2.5995,0.9011,0.4793)$ & 1.1490 \\
5 & $(1.5886,2.0823,0.6572,0.3228)$ & 0.8117 \\
$\vdots$ & $\vdots$ & $\vdots$ \\
19 & $(0.0430,0.1350,0.0048,-0.0130)$ & 0.0302 \\
\hline \hline
\end{tabular}

TABLE 3. Numerical results for the algorithm (4.10)

\section{REFERENCES}

[1] R.P. Agarwal, D. O'Regan and D.R. Sahu, Iterative construction of fixed points of nearly asymptotically nonexpansive mappings, J. Nonlinear Convex Anal. 8(2007), 61-79.

[2] L. Ambrosio, N. Gigli, and G. Savare, Gradient Flows in Metric Spaces and in the Space of Probability Measures, 2nd edn.Lectures in Mathematics ETH Zurich. Birkhäuser, Basel (2008).

[3] D. Ariza-Ruiz, L. Leustean, and G. López, Firmly nonexpansive mappings in classes of geodesic spaces. Trans. Amer. Math. Soc. 366(2014), 4299-4322.

[4] M. Bačák, The proximal point algorithm in metric spaces. Isr. J. Math. 194(2013), 689-701.

[5] H. H. Bauschke, E. Matoušková and S. Reich, Proximal and proximal point methods: convergence results and counterexamples, Nonlinear Anal. 56 (2004), 715-738.

[6] I.D. Berg and I.G. Nikolaev, Quasilinearization and curvature of Alexandrov spaces, Geom. Dedicata 133(2008), 195-218.

[7] M. Bridson and A. Haefliger, Metric Spaces of Nonpositive Curvature, Springer-Verlag, Berlin, Heidelberg, New York, 1999.

[8] F.E. Browder, and W.V. Petrishyn, Construction of fixed points of nonlinear mappings in Hilbert spaces, J. Math. Anal. Appl. 20(1967), 197-228.

[9] K. S. Brown, Buildings, Springer, New York, NY, USA, 1989.

[10] R. E. Bruck and S. Reich, Nonexpansive projections and resolvents of accretive operators in Banach spaces, Houston J. Math. 3 (1977), 459 - 470.

[11] L. C. Ceng, A. R. Khan, Q. H. Ansari and J. C. Yao, Vsicosity approximation method for strongly positive and monotone opterators, Fixed Point Theory 10 (2009), 35 -71.

[12] S. S. Chang, J. C. Yao, L. Wang and L. J. Qin, Some convergence theorems involving proximal point and common fixed points for asymptotically nonexpansive mappings in $C A T(0)$ spaces,Fixed Point Theory Appl. (2016) 2016:68.

[13] R. Chen, and Y. Yao, Strong convergence theorems for strict pseudo-contractions in Hilbert spaces, J. Appl. Math. Comput. 32 (2010), 69-82. 


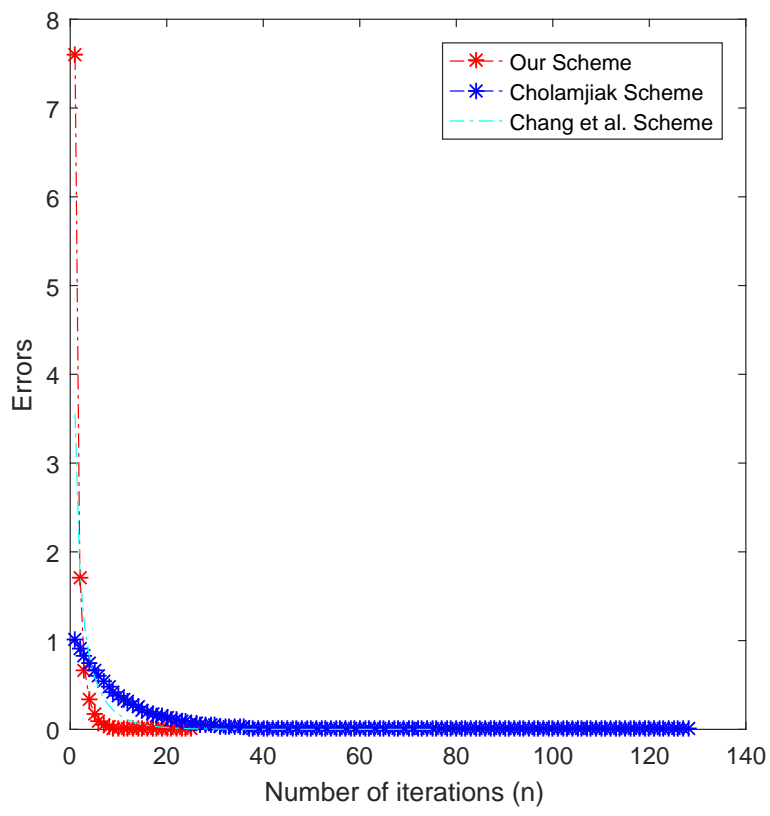

Figure 1. Comparison of errors $\left(\left\|x_{n}-x_{n-1}\right\|_{2}\right)$ in Tables $1-3$

[14] C. E. Chidume and S. Maruster, Iterative methods for the computation of fixed points of demicontractive mappings, J. Comput. Appl. Math. 234 (2010), 861 - 882.

[15] P. Cholamjiak, A. A. Abdou and Y.J. Cho, Proximal point algorithms involving fixed points of nonexpansive mappings in CAT(0) spaces, Fixed Point Theory Appl. (2015)2015:27.

[16] P. L. Combettes and J. C. Pesquet, Proximal splitting methods in signal processing, arXiv:0912.3522v4 [math. OC], (2010).

[17] H. Dehghan and J. Rooin, A characterization of metric projection in CAT(0) spaces, In: International Conference on Functional Equation, Geometric Functions and Applications (ICFGA 2012), 10-12th May 2012, pp. 41-43. Payame Noor University, Tabriz, (2012).

[18] S. Dhompongsa, W.A. Kirk and B. Panyanak, Nonexpansive set-valued mappings in metric and Banach spaces, J. Nonlinear Convex Anal. 8 (2007), 35 - 45.

[19] S. Dhompongsa, W. A. Kirk and B. Sims, Fixed points of uniformly lipschitzian mappings, Nonlinear Anal. 65 (2006), 762 - 772.

[20] S. Dhompongsa and B. Panyanak, On $\triangle$-convergence theorems in $C A T(0)$ spaces, Comput. Math. Appl. 56 (2008), 2572 - 2579.

[21] O. P. Ferreira and P. R. Oliveira, Proximal point algorithm on Riemannian manifolds, Optimization 51 (2002), $257-270$.

[22] A. Genel, and J. Lindenstrauss, An example concerning fixed points. Isr. J. Math. 22 (1975), 81-86.

[23] K. Goebel and S. Reich, Uniform Convexity, Hyperbolic Geometry, and Nonexpansive Mappings, Marcel Dekker, New York and Basel, 1984.

[24] O. Güler, On the convergence of the proximal point algorithm for convex minimization, SIAM J. Control Opt. 29 (1991), 403 - 419.

[25] J. Jost, Convex functionals and generalized harmonic maps into spaces of nonpositive curvature, Comment. Math. Helv. 70(1995), 659-673.

[26] B.A. Kakavandi, Weak topologies in complete CAT(0) metric spaces, Proc. Amer. Math. Soc., 141 (2013), 1029 - 1039. 
[27] N.Hussain, G. Marino, L. Muglia, and B.A.S. Alamri, On some Mann's type iterative algorithms. Fixed Point Theory Appl. (2015)2015:17.

[28] M. A. Khamsi and A. R. Khan, Inequalities in metric spaces with applications, Nonlinear Anal. 74 (2011), 4036 - 4045.

[29] W.A. Kirk, Geodesic geometry and fixed point theory, in Seminar of Mathematical Analysis (Malaga/Seville, 2002/2003), vol. 64 of Colecc. Abierta, pp. 195-225, University of Seville, Secretary Publication, Seville, Spain, 2003.

[30] W.A. Kirk, Geodesic geometry and fixed point theory II, in International Conference on Fixed Point Theory and Applications, pp. 113142, Yokohama Publ., 2004.

[31] W.A. Kirk and B. Panyanak, A concept of convergence in geodesic spaces, Nonlinear Anal. 68 (2008), 3689 - 3696.

[32] T.C. Lim Remarks on some fixed point theorems, Proc. Amer. Math. Soc. 60 (1976), 179-182.

[33] C. Li, G. Lépez and V. Martín Márquez, Monotone vector fields and the proximal point algorithm on Hadamard manifolds, J. London Math. Soc. 79 (2009), 663 - 683.

[34] X. Liu and S. S. Chang, Convergence theorems on total asymptotically demicontractive and hemicontractive mappings in CAT(0) spaces, J. Ineq. Appl. (2014), 2014:436.

[35] P. E. Maingé, Strong convergence of projected subgradient methods for nonsmooth and nonstrictly convex minimization, Set-Valued Anal. 16 (2008), 899 - 912.

[36] G. Marino, B. Scardamaglia1 and E. Karapinar, Strong convergence theorem for strict pseudo-contractions in Hilbert spaces, J. Ineq. Appl. (2016) 2016:134.

[37] G. Marino and H.K. Xu, Weak and strong convergence theorems for strict pseudocontractions in Hilbert spaces, J. Math. Anal. Appl. 329 (2007), 336-346.

[38] B. Martinet, Régularisation d'Inéquations Variationnelles par Approximations Successives, Rev.Franćaise d'Inform. et de Rech. Opérationnelle 3 (1970), 154-158.

[39] U. F. Mayer, Gradient flows on nonpositively curved metric spaces and harmonic maps, Commun. Anal. Geom. 6 (1998), 199-253.

[40] B. Nanjaras and B. Panyanak, Demiclosed principle for asymptotically nonexpansive mappings in CAT(0) spaces, Fixed Point Theory Appl. Vol. 2010, Article ID 268780, 2010,14 pages.

[41] W. R. Mann, Mean value methods in iteration. Proc. Amer. Math. Soc. 4 (1953), 506-510.

[42] S. Reich, Weak convergence theorems for nonexpansive mappings in Banach spaces. J. Math. Anal. Appl. 67 (1979), 274-276.

[43] S. Reich, Strong convergence theorems for resolvents of accretive operators in Banach spaces, J. Math. Anal. Appl. 75 (1980), 287-292

[44] E. A. Papa Quiroz and P. R. Oliveira, Proximal point methods for quasiconvex and convex functions with Bregman distances on Hadamard manifolds, J. Convex Anal. 16 (2009), 49 69.

[45] T. Rockafellar, Monotone operators and the proximal point algorithm, SIAM J. Control Opt. 14 (1976), 877 - 898

[46] R. T. Rockafellar, Characterization of the subdiferentials of convex functions, Pacific J. Math. 17 (1966), 497-510.

[47] N. Shahazad, and H. Zegeye, Approximating common point of fixed points of a pseudocontractive mapping and zeros of sum of monotone mappings, Fixed Point Theory Appl. (2014)2014:85.

[48] S. Kamimura and W. Takahashi, Approximation solutions of maximal monotone operators in Hilbert space, J. Approx. Theory, 106 (2000), 226 - 240.

[49] W. Takahashi et al., A convexity in metric space and nonexpansive mappings, Kodai Mathematical Seminar Reports, 22(1970), 142 - 149.

[50] H. K. Xu, Iterative algorithms for nonlinear operators, J. London Math. Soc., 66(2002), $240-256$.

${ }^{1}$ Department of Mathematics, Michael Okpara University of Agriculture, Umudike, Abia State, Nigeria

E-mail address: ${ }^{1}$ ugwunnadi4u@yahoo.com

${ }^{2}$ Department of Mathematics and Statistics, King Fahd University of Petroleum and Minerals Dhahran 31261 Saudi Arabia.

E-mail address: ${ }^{2}$ arahim@kfupm.edu.sa 
${ }^{3}$ Department of Mathematics and Applied Mathematics, University of Pretoria, Lynnwood Road, Pretoria 0002, South Africa.

E-mail address: ${ }^{3}$ abbas.mujahid@gmail.com 